\title{
MIXTURES OF GAUSSIAN DISTRIBUTIONS UNDER LINEAR DIMENSIONALITY REDUCTION
}

\author{
Ahmed Fawzi Otoom, Oscar Perez Concha and Massimo Piccardi \\ Faculty of Engineering and IT, University of Technology, Sydney (UTS), Sydney, Australia \\ \{afaotoom, oscarpc, massimo\}@it.uts.edu.au
}

Keywords: Dimensionality reduction, Linear transformation, Random projections, Mixture models, Object classification.

\begin{abstract}
High dimensional spaces pose a serious challenge to the learning process. It is a combination of limited number of samples and high dimensions that positions many problems under the "curse of dimensionality", which restricts severely the practical application of density estimation. Many techniques have been proposed in the past to discover embedded, locally-linear manifolds of lower dimensionality, including the mixture of Principal Component Analyzers, the mixture of Probabilistic Principal Component Analyzers and the mixture of Factor Analyzers. In this paper, we present a mixture model for reducing dimensionality based on a linear transformation which is not restricted to be orthogonal. Two methods are proposed for the learning of all the transformations and mixture parameters: the first method is based on an iterative maximum-likelihood approach and the second is based on random transformations and fixed (non iterative) probability functions. For experimental validation, we have used the proposed model for maximum-likelihood classification of five "hard" data sets including data sets from the UCI repository and the authors' own. Moreover, we compared the classification performance of the proposed method with that of other popular classifiers including the mixture of Probabilistic Principal Component Analyzers and the Gaussian mixture model. In all cases but one, the accuracy achieved by the proposed method proved the highest, with increases with respect to the runner-up ranging from $0.2 \%$ to $5.2 \%$.
\end{abstract}

\section{INTRODUCTION}

In the pattern recognition literature, it is widely recognized that high dimensional spaces cause particular difficulties in designing a classifier. One of the reasons is that, in many applications, data points are represented in a high dimensional space when intrinsically they lie in a manifold of much lower dimensionality. Secondly, high dimensions imply high number of parameters which makes the computational task of manipulating and inverting large matrices too expensive. Thirdly, the behaviour and interpretability of concepts acquired for low dimensionality spaces are not always generalized to spaces of many dimensions. Finally, the exponential growth for the need of training samples with the increasing number of dimensions. The combination of all these severe difficulties that can be present in high dimensional spaces is called the "curse of dimensionality" (Bellman, 1961). In order to mollify the curse, classification is often preceded by a dimensionality reduction step where the original features are combined into a significantly smaller set.
One of the simplest and widely applied techniques for this purpose is Principle Component Analysis (PCA). In PCA, the data in the high dimensional space $(P-$ dimensional $)$ are transformed by an orthogonal projection into a lower dimensional space $(D$-dimensional). This transformation is performed in a way that maximizes the variance of the projected data by computing the eigenvalue decomposition of the data covariance matrix. In the reduced space, clustering analysis, density modeling and classification are carried out often with higher classification accuracy than in the original space. PCA has proved to be successful in many applications; however, if the dimensionality of the feature space is very high, the calculation of the eigenvectors becomes computationally hard with a complexity of $O\left(n P^{2}\right)+O\left(P^{3}\right)$, where $n$ is the number of data vectors (often, the $O\left(n P^{2}\right)$ is the dominant term). Moreover, variance maximization may not necessarily lead to high class discrimination. A simple yet interesting alternative approach is to use random transformations (Kaski, 1998), (Fodor, 2002). 
In this approach, the original data $Y$ is transformed into the lower dimensional $X$ via: $X=W Y$, where $W$ is a $D \times P$ matrix $(D<<P)$ and its columns are realizations of independent and identically distributed (i.i.d) zero-mean normal variables, scaled to have unit length (Fodor, 2002). Therefore, the complexity of computing the random matrix is $O(P D)$. It has been shown that projecting the data to a random lowerdimensional subspace yields results comparable to conventional methods such as PCA, as long as the reduced dimension is sufficiently large (Kaski, 1998), (Bingham and Mannila, 2001).

On the other hand, PCA has also been refined as a maximum likelihood solution for a probabilistic latent variable model, commonly known as Probabilistic PCA (PPCA) (Tipping and Bishop, 1999b). In PPCA, a $P$-dimensional observed data vector $y$ can be described in terms of a $D$-dimensional latent vector $x$ as:

$$
y=W x+\mu+\varepsilon
$$

where $W$ is a $P \times D$ matrix describing a linear transformation and $\varepsilon$ is an independent Gaussian noise with a spherical covariance matrix $\sigma^{2} \mathbf{I}$. In this direction, Factor analysis (FA) (Bartholomew, 1987) is closely related to PPCA, except that the noise is assumed to have a diagonal covariance matrix.

In recent years, there have been growing interest in developing different techniques for discovering embedded, locally-linear manifolds of lower dimensionality that extend the above methods including: mixture of PCA (Hinton et al., 1997), MPPCA (Tipping and Bishop, 1999a), and mixture of FA (Ghahramani and Hinton, 1997), amongst others.

In this paper, we present a novel probabilistic mixture model for dimensionality reduction. Each component of the mixture consists of a linear transformation projecting the original data onto a subspace and a Gaussian distribution is fitted on the projected data. This approach is inspired by a sensor fusion analogy, where each component of the mixture is seen as a sensor that can capture a good representation of the original data by finding the best transformation matrix to represent the data into a new reduced space, and then, fitting a Gaussian distribution over the transformed data. For this reason and for immediacy, we have named the proposed method MLiT - mixture of Gaussians under Linear Transformations.

One of the main novelties of our technique is that the transformation matrices are not restricted to be orthogonal, and this paper explores how this will have an effect on the final classification performance. Two different ways are proposed to learn the model's parameters: i. The first approach initializes the transformation matrices to orthogonal base vectors, and then learns the parameters of all the transformation matrices and Gaussian distributions in a maximumlikelihood framework (which might cause the vectors to adopt a non orthogonal arrangement) by using an Expectation-Maximization (EM) algorithm.

ii. The second approach, faster and less computationally expensive than the first one, assigns the transformation matrices to random matrices and fixes the Gaussians based on the sample mean and covariance. According to (Kaski, 1998), in a high dimensional space, there are a much larger number of sufficiently close to orthogonal than orthogonal vectors that might likely be found by carrying out a random mapping.

The proposed technique, MLiT, is used to learn class-conditional likelihoods for maximumlikelihood classification of five "hard" data sets from the UCI repository and the authors' own. Moreover, our model is compared against the well known MPPCA and the conventional Gaussian Mixture Model (GMM).

This paper is organized as follows: Section 2 presents the proposed method (MLiT), the maximum likelihood solution, the initialization procedure for this solution, and the learning of the transformation's parameters using random matrices. Section 3 describes the experiments conducted to evaluate MLiT over multiple data sets, comparing the results with state-of-the-art classifiers. Finally, in section 4, we draw our conclusions and discuss future work.

\section{APPROACH AND METHODOLOGY}

In this section, we describe MLiT, a method for generating a mixture distribution in a dimensionally reduced space that can be useful for density modelling and classification. We first describe the model in the next subsection. We then present in Subsection 2.2 the maximum-likelihood solution devised to learn the model from a set of samples. We also discuss the initialization procedure for this solution. Finally, in Subsection 2.3, we present another way for learning the model's parameters based on random matrices. 


\subsection{Mixture of Gaussians under Linear Transformations (MLiT)}

Let us consider a multivariate random variable, $y$, in a high, $P$-dimensional space. We define the lower, $D$-dimensional space through a compressive linear model

$$
x=\Omega y
$$

where $\Omega$ is a $D \times P$ real matrix, with $D<=P$ and typically $D<<P$. We also posit a density function, $p(x)$, in $x$-space and consider

$$
f(y)=p(\Omega y)=p(x) ;
$$

$f(y)$ is not a proper density in $y$-space: rather, a probability function that repeats the probability density $p(x)$ for all $y$ points satisfying $x=\Omega y$. As such, $f(y)$ expresses the probability of the combination of two distributions: a distribution modelled by $p(x)$ in the $D$-dimensional subspace spanned by the rows of $\Omega$ (the retained dimensions); and a uniform distribution along the $(P-D)$-dimensional subspace satisfying equation $x=\Omega y$ for any given $x$ (the discarded dimensions). For instance, if $p(x)$ is Gaussian, $f(y)$ has the shape of a Gaussian "ridge" i.e. a $D$-dimensional Gaussian function which repeats itself along the direction of $x=\Omega y$ in $y$-space. When referring to its distributional properties hereafter, we will refer to this distribution as Gaussian-uniform. Following the sensor analogy, $x$ can be seen as a view of $y$ made available by a sensor. If the representation power of $x$ is adequate, it will permit to successfully study properties of $y$ e.g. classify measurements in classes of interest.

In general, exploiting an array of $M$ sensors can offer a richer representation of $y$ than a single sensor. By calling $f(y \mid l)$ the probability function for the $l$-th sensor in the array, $l=1 . . M$, it holds that:

$$
f(y \mid l)=p\left(\Omega_{l} y \mid l\right)
$$

where we have assumed that each sensor has its own independent view of $y$, expressed by $\Omega_{l}$ (Kittler, 1998).

Let us now assume that we have a way to estimate a discrete distribution, $p(l)$, stating the quality of the $l$-th sensor at explaining the $y$ sample. From Bayes theorem, we obtain:

$$
f(y, l)=f(y \mid l) p(l)=p\left(\Omega_{l} y \mid l\right) p(l)
$$

By marginalizing over $l$, we obtain the probability function $f(y)$ for the sensor array case:
$f(y)=\sum_{l=1}^{M} f(y, l)=\sum_{l=1}^{M} f(y \mid l) p(l)=\sum_{l=1}^{M} p\left(\Omega_{l} y \mid l\right) p(l)$

which closely recalls the general density of a mixture distribution. However, probabilities are computed in subspaces spanned by linear transformations and such transformations differ for each component. For simplicity of treatment, we further assume that the individual sensor densities are Gaussian, and note $\alpha_{l}=p(l)$, obtaining:

$$
f(y)=\sum_{l=1}^{M} \alpha_{l} \mathcal{N}\left(\Omega_{l} y \mid \mu_{l}, \Sigma_{l}\right)
$$

where the $\mathcal{N}\left(\Omega_{l} y \mid \mu_{l}, \Sigma_{l}\right)$ terms are the densities in the subspaces; means $\mu_{l}$, and covariance matrices $\Sigma_{l}$ are the parameters of each Gaussian component in the $l$-th subspace for $l=1 . . M$; weights $\alpha_{l}$ are the mixing coefficients.

Once an $f(y)$ density is learnt for each $c$ class, $c=1 . . C$, maximum likelihood classification can be simply attained as:

$$
c^{*}=\arg \max _{c}(f(y \mid c))
$$

We note that this model makes no attempt at positioning the subspaces over clusters of data in $y$-space or minimizing reconstruction errors. As such, the number of views is not in correspondence with the number of clusters in the sample set. Rather, each view is justified by a good likelihood fit i.e. providing high within-class invariance.

\subsection{Maximum Likelihood (ML) Solution}

We propose maximum likelihood as one way for jointly finding the parameters $\theta_{l}=\left\{\alpha_{l}, \mu_{l}, \Sigma_{l}, \Omega_{l}\right\}_{l=1}^{M}$. To this aim, we consider a set of i.i.d. observations, $Y=\left\{y_{i}\right\}_{i=1 . . N}$, in the high dimensional space. Our goal is then that of finding values for parameters of (7) maximizing likelihood

$L(\theta)=p(Y \mid \theta)=\prod_{i=1}^{N} f\left(y_{i}\right)=\prod_{i=1}^{N}\left(\sum_{l=1}^{M} \alpha_{l} \mathcal{N}\left(\Omega_{l} y_{i} \mid \mu_{l}, \Sigma_{l}\right)\right)$

where $\theta=\left\{\Omega_{l}, \alpha_{l}, \mu_{l}, \Sigma_{l}\right\}$ and $l=1 . . M$. As usual in similar cases, rather than attempting maximization of (9) directly, we adopt an EM approach. This requires positing the existence of a set of discrete, $M$ valued latent variables, $Z=\left\{z_{i}\right\}_{i=1 . . N}$, whose minimum requirement is that the expression of joint probability function $f\left(y_{i}, Z\right)$ be simpler than $f\left(y_{i}\right)$ itself. 
The target for maximization is the expected value of the complete-data log-likelihood,

$$
Q\left(\theta, \theta^{g}\right)=\sum_{Y}\left[\ln \left(p(Y, Z \mid \theta) p\left(Z \mid Y, \theta^{g}\right)\right]\right.
$$

where $\theta$ and $\theta^{g}$ represent the new and old parameters in the EM iterations, respectively. In (10), $Z$ represents a single realization of the entire set of the latent variables and the summation extends over all its possible $M^{N}$ values. The whole derivation has been presented by the authors in a previous work.

The E-step computes $p\left(z_{i}=l \mid y_{i}, \theta^{g}\right)$, or $p\left(l \mid y_{i}, \theta^{g}\right)$ for brevity, which is the responsibility of the $l$-th component for the $y_{i}$ sample (Bishop, 2006).

$$
p\left(l \mid y_{i}, \theta^{g}\right)=\frac{\alpha_{l}^{g} \mathcal{N}\left(\Omega_{l} y_{i} \mid \mu_{l}^{g}, \Sigma_{l}^{g}\right)}{\sum_{k=1}^{M} \alpha_{k}^{g} \mathcal{N}\left(\Omega_{k} y_{i} \mid \mu_{k}^{g}, \Sigma_{k}^{g}\right)}
$$

Maximizing (10) leads to the following M-step for the parameters:

$$
\begin{gathered}
\alpha_{l}=\frac{1}{N} \sum_{i=1}^{N} p\left(l \mid y_{i}, \theta^{g}\right) \\
\mu_{l}=\frac{\sum_{i=1}^{N} \Omega_{l} y_{i} p\left(l \mid y_{i}, \theta^{g}\right)}{\sum_{i=1}^{N} p\left(l \mid y_{i}, \theta^{g}\right)} \\
\Sigma_{l}=\frac{\sum_{i=1}^{N}\left(\Omega_{l} y_{i}-\mu_{l}\right)\left(\Omega_{l} y_{i}-\mu_{l}\right)^{T} p\left(l \mid y_{i}, \theta^{g}\right)}{\sum_{i=1}^{N} p\left(l \mid y_{i}, \theta^{g}\right)}
\end{gathered}
$$

For the maximization of $\Omega_{l}$, we considered this matrix as $P, D \times 1$ column vectors, $\Omega_{l}=\left(w_{j}\right)_{l}, j=$ $1 . . P$, and we update it column by column, rather than the whole matrix at once. Therefore, the re-estimation formula for $\left(w_{1}\right)_{l}$ is the following:

$$
\begin{aligned}
& \left(w_{1}\right)_{l}= \\
& =\frac{\sum_{i=1}^{N}\left(-\left(w_{2}^{g}\right)_{l} y_{i 2}-\ldots-\left(w_{P}^{g}\right)_{l} y_{i P}+\mu_{l}^{g}\right) y_{i 1} p\left(l \mid y_{i}, \theta^{g}\right)}{\sum_{i=1}^{N} y_{i 1}^{2} p\left(l \mid y_{i}, \theta^{g}\right)}
\end{aligned}
$$

where $N$ is the number of samples, $\left(w_{j}^{g}\right)_{l}, j=2 . . P$ are the other columns" "old" values, $\theta^{g}$ represent the model's old parameters.

Two important issues may occur as a result of the projection step:

i. the component densities across the mixture model and across different classes, can be different in scale. By this, we mean that the linearly transformed space (the $x$-space) does not have a defined scale; therefore, likelihood $p(x)$ can be made arbitrarily larger or smaller by changes to the scale of $x$. ii. as a consequence of this and the maximumlikelihood target, the scale of $x$ may tend to 0 along iterations to endorse high values of $p(x)$. In turn, this implies that the projection matrix may also tend to zero (an undesirable solution that we call degenerate or singular hereafter).

In order to avoid these problems, we propose the normalization of the projection matrix at each step of the EM algorithm; henceforth, we refer to this method as MLiT (Normalized) or MLiT (N). By equating the concept of norm to that of scale, this will make the densities of equal scale across the different components, and also across different classes. Further, this will avoid $\Omega_{l}$ reaching the degenerate solution and act as a likelihood regularization. Therefore, after each EM step, we normalize $\Omega_{l}$ as follows:

$$
\Omega_{l}=\frac{\Omega_{l}}{\operatorname{Norm}\left(\Omega_{l}\right)}
$$

We have tried several norms (L1, L2, Infinity and Frobenius), with the Frobenius norm providing the highest and most stable results. Therefore, in the following, we report results based on this norm:

$$
\text { Frobenius_norm }\left(\Omega_{l}\right)=\sqrt{\sum \operatorname{diag}\left(\Omega_{l}^{T} \Omega_{l}\right)}
$$

Thus, MLiT (N) searches for possible solutions over the likelihood space. Every time a solution is provided by the maximization step of EM, we normalize the projection matrix $\Omega_{l}$ in order to keep it on an equal scale. The expectation-maximization steps become therefore expectation-maximizationnormalization steps. An obvious disadvantage of this approach is that the new normalized solution might or might not have higher likelihood than the previous normalized solution. For this reason, we monitor the evolution of the likelihood along the iterations and elicit ad-hoc convergence criteria.

\subsubsection{Initialization Phase}

In EM, the parameter values traversed along the iterations and the likelihood value achieved at convergence may strongly depend on the parameters' initial values. For our approach, we choose to apply a deterministic initialization to ensure repeatable results at each run. Namely, we decided to initialize the projection matrix, $\Omega$, by the orthonormal transformation provided by PCA, selecting either the largest or the smallest eigenvectors (i.e. the eigenvectors associated with the largest and smallest eigenvalues, respectively).

Projecting the data by the largest eigenvectors transforms them into a space where their variance 
is maximized and, under the hypothesis that their distribution be Gaussian, the likelihood is minimum amongst all orthonormal projections (Bolton and Krzanowski, 1999), and therefore forcing the EM to explore a large region of the parameter space before convergence. Conversely, projecting them with the smallest eigenvectors transforms them into a space where their variance is minimized and likelihood is maximum.

In our experiments, we noticed that there is no certain initialization method that always provide the best classification accuracy. Thus, we experiment with both methods and choose the one providing better accuracy results.

As the data per class are projected to each of the components, the remaining initial parameters of the EM algorithm are chosen as follows:

- The initial mean $\mu_{l}$ and covariance matrix $\Sigma_{l}$ of each component will be the sample mean and covariance computed directly from the projected data of each component, $x_{l}$ :

$$
\begin{aligned}
& -\mu_{l}=\frac{1}{N} \sum_{n=1}^{N} x_{l n} \\
& -\Sigma_{l}=\frac{1}{N-1} \sum_{n=1}^{N}\left(x_{l n}-\mu_{l}\right)\left(x_{l n}-\mu_{l}\right)^{T}
\end{aligned}
$$

- The initial priors $\alpha_{l}$ for $l=1$...M, are chosen to be equal across all the components:

$$
-\alpha_{l}=\frac{1}{M}
$$

\subsection{Random Transformations Solution}

In this subsection, we present another method for learning the transformation matrix, $\Omega_{l}$, based on using random matrices (MLiT $(\mathrm{R}))$. The main idea is to use random matrices of Gaussian distributed elements and with unit length columns. This idea is motivated by the Johnson-Lindenstrauss lemma (Johnson and Lindenstrauss, 1984): if points in a feature space $Y$ ( $P$-dimensional $)$ are projected onto a randomly selected subspace of suitable high dimension $D$, then the distances between the points are approximately preserved if $D$ is large enough.

$$
\begin{aligned}
& <\left(\left\|\phi\left(y_{i}\right)-\phi\left(y_{j}\right)\right\|_{D}^{2}-\left\|y_{i}-y_{j}\right\|_{P}^{2}\right)^{2}>_{\phi} \leq \\
& \quad \leq \frac{2}{D}\left\|\left(y_{i}\right)-\left(y_{j}\right)\right\|_{P}^{4}
\end{aligned}
$$

where \|\|$_{P}$ and \|\|$_{D}$ denote the Euclidean distance norms in $V_{P}$ and $V_{D}$, respectively, and $<>_{\phi}$ is the average over all possible isotropic random choices for the unit vectors defining the random mapping $\phi$.

In our case, we chose the elements of the $\Omega_{l}$ to be drawn from a zero-mean normal distribution with a variance of $1 / \sqrt{P}$, where $P$ is the original space dimension. The columns of $\Omega_{l}$ are then normalized to be unitary vectors. Moreover, we decided to have the same transformation matrices across all classes. Thus, with the above settings, the scale of the transformation matrices is comparable.

After transforming the data of each class as: $X_{l}=$ $\Omega_{l} Y$, we fix a Gaussian distribution over the transformed data and the mixture parameters are as follows:

- The priors $\alpha_{l}$ for $l=1 . . M$, are chosen to be equal across all the components.

- The final mean $\mu_{l}$ and covariance matrix $\Sigma_{l}$ of each component are the sample mean and covariance, computed directly from the projected data of each component, $x_{l}$.

\section{EXPERIMENTS AND ANALYSIS}

The empirical evaluation of a classifier's accuracy requires extensive testing over multiple data sets and a comparative analysis with existing, state-of-the-art classifiers. To this aim, in this section we present details on the data sets used and experiments conducted.

\subsection{Data Sets}

We evaluate the proposed method on five data sets, four of which are selected from the UCI Machine Learning Repository (Asuncion and Newman, 2007), and are widely used by the pattern recognition community for evaluating learning algorithms. These four data sets are the Vehicle data set, Wisconsin Diagnostic Breast Cancer data set (WDBC), Wisconsin Prognostic Breast Cancer (WPBC) data set, and Optical Handwritten Digits data set (OpticDigit). The last data set, named Public Premises Video Surveillance data set (PPVS), was collected by the authors themselves.

The Vehicle data set involves classification of a given silhouette as one of four types of vehicles, namely, "bus", "Opel", "Saab" and "van". The vehicle silhouettes are described by various shape measurements. The rationale for choosing this data set is that it is the most similar in the UCI repository to our own data set and can offer a comparative insight into the method's performance. The WDBC and WPBC data sets contain various shape features from images of fine needle aspirates (FNA) of breast mass for diagnosis and prognosis of breast cancer. The OpticDigit data set is based on rescaled bitmaps of handwritten digits: the original $32 \times 32$ black and white bitmaps are divided into non-overlapping blocks of $4 \times 4$ pixels and the number of 'on' pixels counted in each block, 
Table 1: Comparative summary of the data sets used.

\begin{tabular}{cccc}
\hline \hline Data set & \# Features & \# Instances & \# Classes \\
\hline Vehicle & 18 & 846 & 4 \\
OpticDigit & 64 & 5620 & 10 \\
WDBC & 30 & 569 & 2 \\
WPBC & 33 & 198 & 2 \\
PPVS & 44 & 600 & 4 \\
\hline
\end{tabular}

resulting in a 64-dimensional feature vector of homogeneous features. The Public Premises Video Surveillance data set (PPVS) is based on video footage provided by an industrial partner. It involves classification of an object in a video surveillance environment into one of four classes: "trolleys", "bags", "single persons", and "groups of people". The images of these objects have been clipped from video footage acquired at a number of airports and train stations world-wide. The feature set consists of statistics of various local features such as line segments, circles, corners, and global shape descriptors such as fitted ellipses and bounding boxes. This feature set is described in detail in (Otoom, 2007).

As we can conclude from the previous paragraphs and the data displayed in Table 1, there are major differences between these five data sets in terms of the nature of data and application context, number of instances available, number of features extracted, types of features used for representation and number of classes. Therefore, the chosen data sets offer a suitable basis for comparative analysis.

\subsection{Experiments}

In this subsection, we present classification results for the proposed method on the five aforedescribed data sets. We compare the performance of our approach with that of mixture of PPCA (MPPCA) and Gaussian mixture model (GMM). Experiments with these classifiers were carried out in MATLAB by setting all tunable parameters in the most genuine way to achieve the highest performance. We summarize below the main parameters, and in Table 2, we report the values that achieved the best accuracy results. The parameters are as follows:

- GMM: There is one main parameter, the number of the GMM components $(M)$.

- MPPCA: There are two main parameters, the number of reduced dimensions $(D)$, and the number of mixture components $(M)$.

For MLiT, the parameters to adjust are selected as follows:
- The number of the mixture components $(M)$ and reduced dimensions $(D)$ were manually selected as reported in Table 2 .

\section{- For MLiT (N):}

- The initial transformation matrices for each class, $\Omega^{[0]}$, were computed by using either the smallest or the largest consecutive eigenvectors of the covariance matrix of the original data. For example, in the case of largest eigenvectors, two components per class $(M=2)$, and a reduced space of three dimensions $(D=3)$, we select the three first eigenvectors for $\Omega_{1}^{[0]}$ and the eigenvectors between the third and the fifth for $\Omega_{2}^{[0]}$.

- Initial transformed data: $X_{l}^{[0]}=\Omega_{l}^{[0]} Y, l=1 . . M$.

- Initial means, $\mu_{l}^{[0]}$, and variances, $\Sigma_{l}^{[0]}, l=1 . . M$ : from the initial transformed data.

- Equal initial priors for all components, $\alpha_{l}^{[0]}=$ $\frac{1}{M}, l=1 . . M$.

- For MLiT (R):

- $\Omega_{l}$ is chosen randomly from a $1-\mathrm{D}$ zero-mean Gaussian distribution with a variance of $1 / \sqrt{P}$.

- The priors $\alpha_{l}$ for $l=1 . . M$, are chosen to be equal across all the components: $\alpha_{l}=\frac{1}{M}$.

- The mean $\mu_{l}$ and covariance matrix $\Sigma_{l}$ of each component are the sample mean and covariance, computed directly from the projected data of that component, $x_{l}$.

As stopping criteria, for MLiT (N) the normalization step does not guarantee a monotonic increase in the likelihood; hence, we elicit an ad-hoc criteria for stopping by running the EM algorithm for 50 iterations and choosing that delivering the maximum accuracy by cross-validation. For MPPCA, we observed that the accuracy stabilized after 200 iterations. For GMM, instead, accuracy stabilization was empirically achieved after 50 iterations. For validation, we have chosen 5-fold cross-validation since it offers a good trade off between the large bias of the hold-out method and the large variance of the leave-one-out method (Breiman and Spector, 1992). This implies randomly partitioning the data set into five disjoint subsets, training the classifier with four and using the last for testing. Classification accuracy is averaged over five runs by using, in turn, each fold for testing. We express classification accuracy simply as the percentage of correctly classified instances with respect to their total number:

$$
\text { accuracy }=\frac{\text { number of correctly classified samples }}{\text { total number of samples }}
$$


Table 2: Results for 5-fold CV in terms of accuracy (\%) and standard deviation, on five data sets and across different classifiers, with the highest indicated in boldface font. For each dataset, the first row presents the main parameters' values for different classifiers, and the second row shows the achieved accuracy (\%).

\begin{tabular}{|c|c|c|c|c|}
\hline Classifier & $\operatorname{MLiT}(\mathrm{N})$ & $\operatorname{MLiT}(\mathrm{R})$ & MPPCA & GMM \\
\hline $\begin{array}{c}\text { Parameters } \\
\text { Dataset }\end{array}$ & M & M & M & M \\
\hline PPVS & 33 & 30 & 25 & 2 \\
\hline$(\%)$ & $76.7 \pm 2.4$ & $78.5 \pm 2.0$ & $73.5 \pm 4.0$ & $73.3 \pm 2.1$ \\
\hline Vehicle & 14 & 18 & 10 & 2 \\
\hline$(\%)$ & $85.6 \pm 1.9$ & $84.3 \pm 2.1$ & $83.6 \pm 1.3$ & $82.8 \pm 1.9$ \\
\hline OpticDigit & 29 & 35 & 16 & 2 \\
\hline$(\%)$ & $98.4 \pm 0.3$ & $98.3 \pm 0.3$ & $98.6 \pm 0.4$ & $96.9 \pm 0.3$ \\
\hline WDBC & $18 \quad 1$ & $20 \quad 4$ & 20 & 2 \\
\hline$(\%)$ & $96.1 \pm 1.7$ & $95.9 \pm 1.9$ & $94.7 \pm 1.7$ & $95.9 \pm 1.8$ \\
\hline WPBC & 4 & & 15 & 4 \\
\hline$(\%)$ & $77.4 \pm 1.1$ & $76.9 \pm 1.8$ & $76.9 \pm 0.0$ & $75.9 \pm 1.4$ \\
\hline
\end{tabular}

It is important to note that, in the following, we report the classification results in terms of two statistical measures: average accuracy over the various runs, and standard deviation. However, we chose the average accuracy as the main measure for comparing the different classifiers; nevertheless, the standard deviation is an important measure for the precision of the classification accuracy, and it can be considered together with the accuracy for a better estimate of the classification performance.

Table 2 reports the best results of 5-fold crossvalidation on the various data sets and across the compared classifiers. We note that in this table, for MLiT $(\mathrm{N})$, all results are obtained with largest eigenvectors initialization except the cases of Vehicle and WPBC data sets. It is clear from this table that, in all cases (except the PPVS data set), MLiT (N) has slightly outperformed the performance of MLiT (R), proving that the maximum likelihood solution can be a better learning method in comparison to that of learning based on random matrices. However, the margin of improvements is not very high, which indicates that the random transformations solution can deliver promising results with less computation. We can also note from Table 2 that the performance of MLiT outperformed that of MPPCA on four out of the five compared data sets with improvements ranging from $0.5 \%$ to $5.0 \%$, proving the strength of MLiT against a state-of-the-art classifier (MPPCA has slightly outperformed MLiT by $0.2 \%$ only on the OpticDigit data set). Moreover, we can note that, in all cases, the performance of MLiT outperformed that of GMM with improvements ranging from $0.2 \%$ to $5.2 \%$. This illustrates the ability of MLiT in overcoming the curse of dimensionality and providing better performance in the reduced space. MPPCA has also provided better classification results than GMM on majority of the data sets.

Overall, the experiments on the five data sets presented in this section showed that MLiT reported higher experimental accuracy over both compared classifiers (except the case of OpticDigit where MPPCA slightly outperformed MLiT). Interpretation of accuracy results in high dimensional spaces is not immediate. In the case of the ML solution, we lean to attribute these improvements in accuracy to the Gaussian-uniform distribution property of focussing on invariant features. This permits the building of compact models that have proved discriminative when used with the Bayes inversion rule, while it introduces elements of robustness since outliers are ousted to the discarded dimensions during training as much as possible. The non-orthogonality of the transformation adds further degrees of freedom to the model. However, this feature seems to be used only to a limited extent since the maximum accuracy is often achieved during the very first iterations of EM, when the transformation only mildly deviates from orthogonality. In these terms, both the maximum likelihood and random solutions are often close to orthogonal transformations.

\section{CONCLUSIONS}

In this paper, we have presented a method for linear dimensionality reduction within mixture distributions. The model that we have proposed for the classconditional likelihood is a mixture of Gaussian distributions under linear transformations (7). This model 
equates to a uniform distribution along the discarded dimensions and a full Gaussian model along the retained dimensions.

It is important to contrast this model properly to the several existing methods for linear dimensionality reduction in mixture models such as mixtures of PCA, PPCA, and FA. One of the main points of difference is that the linear transformation is not restricted to be orthogonal. Further, the linear model adopted, $x=\Omega y$, does not assume additive noise models and makes $x$ observable. On the ground of that, we can evaluate density $\mathcal{N}(\Omega y \mid \mu, \Sigma)=\mathcal{N}(x \mid \mu, \Sigma)$ directly in $x$-space. For learning the model, we have presented two different methods; the first method learns the model's parameters in a maximum likelihood framework (MLiT (N)). Normalization is proposed as a way to regularize this solution. Thus, a common scale is imposed to all the transformations and a singularity problem is avoided. Another simple yet powerful method for learning the model's parameters can be based on random matrices (MLiT (R)). This method has offered promising and computationally feasible results. However, the maximum likelihood solution delivered better accuracy results in majority of the data sets suggesting that it can be a better way for learning the model's parameters.

The experimental performance of MLiT has proved to outperform that of MPPCA and GMM in almost all cases with improvements ranging from $0.2 \%$ to $5.2 \%$ compared to the runner-up. The only case where MLiT did not deliver the best accuracy is on the OpticDigit data set where it was slightly outperformed by MPPCA by $0.2 \%$. In addition to visual object classification, the proposed method permits general application for density modeling and classification of other continuous numerical data requiring dimensionality reduction. Moreover, its re-estimation formulas can be easily extended to suit boosting and other weighted maximum likelihood targets and adapt to a variety of pattern recognition frameworks.

\section{ACKNOWLEDGEMENTS}

The authors wish to thank the Australian Research Council and iOmniscient Pty Ltd that have partially supported this work under the Linkage Project funding scheme - grant LP0668325.

\section{REFERENCES}

Asuncion, A. and Newman, D. (2007). UCI machine learning repository.

Bartholomew, D. J. (1987). Latent Variable Models and Factor Analysis. Charles Griffin \& Co. Ltd., London.

Bellman, R. (1961). Adaptive control processes - A guided tour. Princeton University Press, Princeton, New Jersey.

Bingham, E. and Mannila, H. (2001). Random projection in dimensionality reduction: applications to image and text data. In Proceedings of the 7th ACM SIGKDD International Conference on Knowledge Discovery and Data Mining (KDD-2001), pages 245-250.

Bishop, C. M. (2006). Pattern Recognition and Machine Learning. Springer.

Bolton, R. J. and Krzanowski, W. J. (1999). A characterization of principal components for projection pursuit. The American Statistician, 53(2):108-109.

Breiman, L. and Spector, P. (1992). Submodel selection and evaluation in regression: The $\mathrm{x}$-random case. International Statistical Review, 60(3):291-319.

Fodor, I. (2002). A survey of dimension reduction techniques. Technical Report UCRL-ID-148494, Lawrence Livermore National Laboratory.

Ghahramani, Z. and Hinton, G. (1997). The EM algorithm for mixtures of factor analyzers. Technical Report CRG-TR-96-1, University of Toronto.

Hinton, G. E., Dayan, P., and Revow, M. (1997). Modeling the manifolds of images of handwritten digits. IEEE Transactions on Neural Networks, 8(1):65-74.

Johnson, W. B. and Lindenstrauss, J. (1984). Extensions of lipschitz mappings into a hilbert space. In Conference in modern analysis and probability, Contemporary Math, volume 26, pages 189-206.

Kaski, S. (1998). Dimensionality reduction by random mapping: Fast similarity computation for clustering. In Proceedings of IJCNN'98, International Joint Conference on Neural Networks, volume 1, pages 413418. IEEE Service Center.

Kittler, J. (1998). Combining classifiers: A theoretical framework. Pattern Analysis and Applications, 1(1):18-27.

Otoom, A. e. a. (2007). Towards automatic abandoned object classification in visual surveillance systems. In Asia-Pacific Workshop on Visual Information Processing, pages 143-149, Tanian, Taiwan.

Tipping, M. E. and Bishop, C. M. (1999a). Mixtures of probabilistic principal component analyzers. Neural Computation, 11(2):443-482.

Tipping, M. E. and Bishop, C. M. (1999b). Probabilistic principal component analysis. Journal of the Royal Statistical Society: Series B (Statistical Methodology),, 61(3):611-622. 


$\begin{array}{ll}\text { From: } & \text { VISAPP Secretariat } \\ \text { To: } & \text { afaotoom@it.uts.edu.au } \\ \text { Subject: } & \text { VISAPP 2010: Author Notification } \\ \text { Date: } & \text { Thursday, 28 January 2010 4:34:29 AM }\end{array}$

Dear Mr. Ahmed Otoom,

We are happy to inform you that the regular paper you have submitted to VISAPP, with number 191, entitled 'Mixtures of Gaussian Distributions under Linear Dimensionality Reduction', has been accepted for a poster presentation (Poster).

All reviews performed by the program committee are now available at the PRIMORIS Author's Home (www.insticc.org/Primoris/). Please login and then click on Author's home / Paper Reviews, to access the reviews.

It is very important that you try to follow the suggestions indicated in the reviews during the preparation of the camera-ready manuscript.

Furthermore, it is EXTREMELY important that you follow the camera-ready paper format and preparation guidelines for the proceedings, which are available at the VISAPP web site http://www.visapp.visigrapp.org//Paper Templates.htm.

Any non-conformance with the specified format may force the proceedings editing team to return the paper to you for re-formatting, and in case of repeated problems it may prevent the paper from being published altogether.

Please note that the publication of any paper in the conference proceedings requires that at least one of the authors is registered until next February 10th, 2010.

Should you have any question regarding the camera ready submission and registration, please don't hesitate to contact the secretariat.

Best regards,

Bruno Encarnação

VISAPP Secretariat 
Review \#: 1

Abstract and Introduction are

Too

adequate?

short

Conclusions/Future Work are convincing?

No

Figures are Adequate?

Improve critical discussion?

in number and quality

Yes

Improve English?

validation

Yes

No

Needs comparative evaluation?

Yes

Needs more experimental results?

Yes

Originality

Newness of the ideas expressed

3

Overall rating

Weighted value of above items

2

Paper formatting needs adjustment?

No

Presentation

Structure/Length/English

3

References are up-to-date and appropriate?

Yes

Relevance

Paper fits one or more of the topic areas?

Is the problem worth the given attention?

Significance

Technical Quality

Theoretical soundness/methodology

Scale: 1:Lowest Value;6:Highest Value

The proposed approach seems interesting although i think more work needs to be done.I would appreciate to have more details and a more precise critical analysis.

Review \#: 2

Abstract and Introduction are

Too adequate?

short

Conclusions/Future Work are convincing?

No

Figures are Adequate?

Improve critical discussion?

in number and quality

Yes

Improve English?

validation

Yes

No

Needs comparative evaluation?

Yes 
Needs more experimental results?

Yes

Originality

Newness of the ideas expressed

3

Overall rating

Weighted value of above items

2

Paper formatting needs adjustment?

Presentation

Structure/Length/English

No

References are up-to-date and appropriate?

Relevance

Paper fits one or more of the topic areas?

4

Yes

Significance

Is the problem worth the given

attention?

Technical Quality

Theoretical soundness/methodology

4

Scale: 1:Lowest Value;6:Highest Value

Given the learning-theoretical (rather than computer vision-theoretical) nature of this paper, I do not find it suitable for this venue: a machine learning/pattern recognition conference would be more appropriate. The proposed approach seems interesting although its practical implementation seems ridden with some difficulties (esp. method $\mathrm{N}$ ). Given the marginal improvements presented, the difficulties do not seem worth the effort. Since the proposed method is a method of dimension reduction, test sets with higher dimensions should be used than the ones presented in the paper. Also a comparison should be presented with regular SVMs (applied to the original data without dimension reduction) to get a better reference for the meaning of the reported improvements. The discussion at (5) and (6) could be improved; Bayes seems to mentioned unnecessarily (simple conditional probability) and a more careful discussion of using basic probability on improper densities would be helpful. 


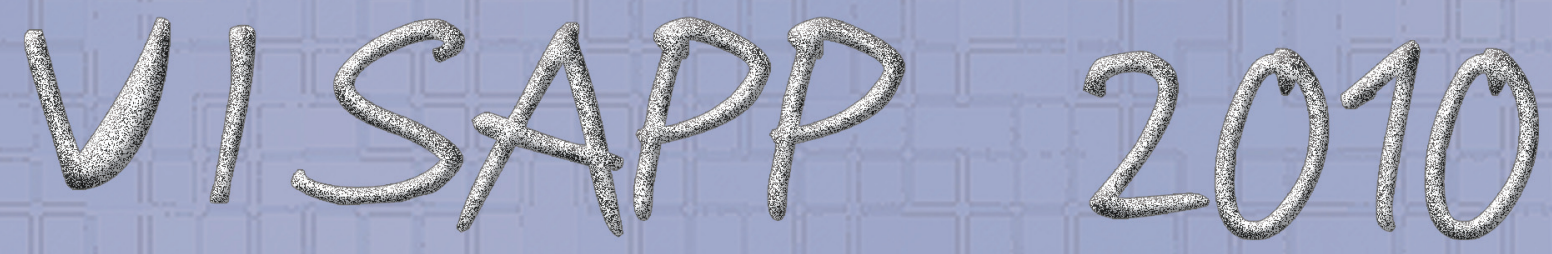

International Conference on Computer Vision Theory and Applications

\section{Proceedings}

Volume 2

Angers, France 17 - 21 May, 2010 


\title{
VISAPP 2010
}

\author{
Proceedings of the \\ International Conference on \\ Computer Vision Theory and Applications
}

Volume 2

Angers, France

May $17-21,2010$

Organized by

INSTICC - Institute for Systems and Technologies of Information, Control and Communication

Locally Organized and Hosted by

UA - Université d'Angers

IstiA - École d'ingénieurs de l'Université d'Angers

LISA - Laboratoire d'Ingénierie des Systèmes Automatisés

Technical Co-sponsorship by

WfMC - Workflow Management Coalition 
Copyright (c) 2010 INSTICC - Institute for Systems and Technologies of Information, Control and Communication

All rights reserved

Edited by Paul Richard and José Braz

Printed in Portugal

ISBN: 978-989-674-029-0

Depósito Legal: 308933/10

http://www.visapp.visigrapp.org/

visapp.secretariat@insticc.org 


\section{Organizing AND Steering Committees}

\section{CONFERENCE CO-CHAirs}

José Braz, Escola Superior de Tecnologia de Setúbal, Portugal

Paul Richard, Laboratoire D’ingénierie des Systèmes Automatisés - LISA, France

\section{Proceedings Production}

Helder Coelhas, INSTICC, Portugal

Andreia Costa, INSTICC, Portugal

Bruno Encarnação, INSTICC, Portugal

Bárbara Lima, INSTICC, Portugal

Raquel Martins, INSTICC, Portugal

Liliana Medina, INSTICC, Portugal

Carla Mota, INSTICC, Portugal

Vitor Pedrosa, INSTICC, Portugal

Filipa Rosa, INSTICC, Portugal

José Varela, INSTICC, Portugal

\section{CD-ROM PRODUCTION}

Elton Mendes, INSTICC, Portugal

Pedro Varela, INSTICC, Portugal

\section{Graphics Production and Webdesigner}

Daniel Pereira, INSTICC, Portugal

\section{SECRETARIAT}

Bruno Encarnação, INSTICC, Portugal

\section{WEBMASTER}

Sérgio Brissos, INSTICC, Portugal 


\section{AUXiliary ReVieWERS}

Maria Andersson, FOI Swedish Defence Research Agency, Sweden

Beatriz Andrade, Universidade Federal do Paraná, Brazil

Florent Brunet, Université d'Auvergne, France

Alan Brunton, University of Ottawa, Canada

Christina Grönwall, Defence Research Establishment, Sweden

Xin Li, Princeton University, U.S.A.

Henry Medeiros, Purdue University, U.S.A.

Mirko Meuter, Delphi Electronics and Safety, Germany
Christian Nunn, University of Wuppertal, Germany

Giovanni Puglisi, University of Catania, Italy

Maurício Pamplona Segundo, Imago Research Group, Brazil

Jasmine seng, Nottingham University Malaysia Campus, Malaysia

Roberto Toldo, University of Verona, Italy

Stefanie Wuhrer, National Research Council of Canada, Canada

Yinghai Zhao, University of Science and Technology of China, China

\section{Selected Papers Book}

A number of selected papers presented at VISAPP 2010 will be published by Springer-Verlag in a CCIS Series book. This selection will be done by the Conference Co-chairs, among the papers actually presented at the conference, based on a rigorous review by the VISIGRAPP 2010 Program Committee members. 


\section{FOREWORD}

This volume contains the proceedings of the Fifth Edition of International Conference on Computer Vision Theory and Applications (VISAPP 2010), organized by the Institute for Systems and Technologies of Information, Communication and Control (INSTICC) and Technical Co-Sponsorship by the Workflow Management Coalition(WfMC).

The field of imaging and modelling overlaps the research areas of computer vision and computer graphics. Applications are found in different areas like robotics, industrial design, computer vision, computer animation, human factors and engineering, video surveillance, medicine, visual communications, and imaging sciences in general. Since its first edition in 2006, VISAPP has been bringing together researchers from all over the world to share new knowledge, insights, and experimental results in these areas. The conference focused on four different areas related to computer vision, from Image Processing to Image Understanding including Image Analysis and Stereo Vision. All tracks focus on real world applications and highlight the benefits of computer vision for industry and services, thus making a bridge between the Academia and the Enterprise worlds.

VISAPP 2010 received 260 paper submissions from more than 50 countries in all continents. From these, 34 papers were published and presented as full papers, 70 were accepted for short presentation and another 48 for poster presentation. These numbers, leading to a fullpaper acceptance ratio of $13 \%$, and a total oral acceptance ratio of $40 \%$, shows the intention of preserving a high quality forum for the next editions of this conference. Additionally, in this edition of VISAPP, a workshop was organized which facilitated the presentation of highly focused work in specialized themes within the topics of conference. Moreover, the conference also featured a number of keynote lectures delivered by internationally well known experts thus contributing to increase the overall quality of the conference and to provide a deeper understanding of the conference interest fields.

The high number and high quality of the papers received imposed difficult choices in the review process. Each paper was sent to at least three reviewers. From these reviews, those papers that were adequately balanced in terms of quality, originality, and relevance to the conference themes were selected. A short list of the papers presented at the conference will be selected and extended revised versions of them will be included in a book to be published by Springer-Verlag. Two awards are delivered at the closing session, to recognize the best conference paper and the best student paper. The conference success is due to the efforts of many people.

We gratefully acknowledge the essential contributions of VISAPP Program Committee members and additional reviewers in evaluating the papers and also special thanks to all the members of the INSTICC organizing committee. 
We wish you all an exciting conference and an unforgettable stay in Angers. We hope to meet you again for the 6th edition of VISAPP conference details of which will be shortly available at http://www.visapp.visigrapp.org.

\section{Paul Richard}

Laboratoire D’ingénierie Des Systèmes Automatisés - LISA, France

\section{José Braz}

Escola Superior de Tecnologia de Setúbal, Portugal 


\section{CONTENTS}

\section{INVITED SPEAKERS}

\section{KEYNOTE SPEAKERS}

INVERSE PROBLEMS IN IMAGING AND COMPUTER VISION - From Regularization Theory to Bayesian Inference

Ali Mohammad-Djafari

TWO NEW APPROACHES TO DEPTH OF FIELD POSTPROCESSING

Brian A. Barsky

IS-9

MODELING DEFORMABLE SURFACES FROM SINGLE VIDEOS

Pascal Fua

IS-19

FISHER KERNEL REPRESENTATION OF IMAGES AND SOME OF ITS SUCCESSFUL APPLICATIONS

IS-21

Gabriela Csurka

\section{IMAGE ANALYSIS}

\section{Full Papers}

HYPERACCURATE ELLIPSE FITTING WITHOUT ITERATIONS

Kenichi Kanatani and Prasanna Rangarajan

TREE-STRUCTURED TEMPORAL INFORMATION FOR FAST HISTOGRAM COMPUTATION

Séverine Dubuisson

CIRCLE DETECTION USING THE IMAGE RAY TRANSFORM - A Novel Technique for using a Ray Analogy to Extract Circular Features Alastair H. Cummings, Mark S. Nixon and John N. Carter

MINIMUM SPANNING TREE FUSING MULTI-SALIENT POINTS HIERARCHICALLY FOR MULTI-MODALITY IMAGE REGISTRATION

Shaomin Zhang, Lijia zhi, Dazhe Zhao and Hong Zhao

MULTISPECTRAL TEXTURE ANALYSIS USING LOCAL BINARY PATTERN ON TOTALLY ORDERED VECTORIAL SPACES

Vincent Barra

FACE DETECTION AND TRACKING WITH 3D PGA CLM

Meng Yu and Bernard Tiddeman

MOTION SEGMENTATION OF ARTICULATED STRUCTURES BY INTEGRATION OF VISUAL PERCEPTION CRITERIA

Hildegard Kuehne and Annika Woerner

SETTING GRAPH CUT WEIGHTS FOR AUTOMATIC FOREGROUND EXTRACTION INWOOD LOG IMAGES

Enrico Gutzeit, Stephan Ohl, Arjan Kuijper, Joerg Voskamp and Bodo Urban

GRAPH CUTS AND APPROXIMATION OF THE EUCLIDEAN METRIC ON ANISOTROPIC GRIDS

Ondřej Daněk and Pavel Matula 
PATTERN RECOGNITION FOR FAULT DIAGNOSIS OFSOLAR POWER INVERTER BY TRAJECTORY IMAGE UNDERSTANDING

JaeHo Hwang, Nanhwa Kim, Naejoung Kwak and WonPyo Hong

THE POTENTIAL OF CONTOUR GROUPING FOR IMAGE CLASSIFICATION

Christoph Rasche

VISUAL PITCH CLASS PROFILE - A Video-based Method for Real-time Guitar Chord Identification

M. Cicconet, P. Carvalho, L. Velho and M. Gattass

USING SR-TREE IN A CONTENT-BASED AND LOCATION-BASED IMAGE RETRIEVAL SYSTEM

Hien Phuong Lai, Nhu Van Nguyen, Alain Boucher and Jean-Marc Ogier

NON-PARAMETRIC BAYESIAN ALIGNMENT AND RECOVERY OF OCCLUDED FACE USING DIRECT COMBINED MODEL

Ching-Ting Tu and Jenn-jier James Lien

FACIAL POSE ESTIMATION USING ACTIVE APPEARANCE MODELS AND A GENERIC FACE MODEL

Thorsten Gernoth, Katerina Alonso Martínez, André Gooßen and Rolf-Rainer Grigat

FAST NON-LINEAR NORMALIZATION ALGORITHM FOR IRIS RECOGNITION Wen-Shiung Chen, Jen-Chih Li, Ren-He Jeng, Lili Hsieh and Sheng-Wen Shih

MIXTURES OF GAUSSIAN DISTRIBUTIONS UNDER LINEAR DIMENSIONALITY REDUCTION

Ahmed Otoom, Oscar Perez Concha and Massimo Piccardi

AN EVALUATION OF LOCAL IMAGE FEATURES FOR OBJECT CLASS RECOGNITION Saiful Islam and Andrzej Sluzek

TOWARDS DETECTING PEOPLE CARRYING OBJECTS A Periodicity Dependency Pattern Approach

Tobias Senst, Rubén Heras Evangelio, Volker Eiselein, Michael Pätzold and Thomas Sikora

A GENERIC CONCEPT FOR OBJECT-BASED IMAGE ANALYSIS

André Homeyer, Michael Schwier and Horst K. Hahn

HIERARCHICAL OBJECT CLASSIFICATION USING IMAGENET DOMAIN ONTOLOGIES Haider Ali

SUPPRESSION OF UNCERTAINTIES AT EMOTIONAL TRANSITIONS - Facial Mimics Recognition in Video with 3-D Model

Gerald Krell, Robert Niese, Ayoub Al-Hamadi and Bernd Michaelis

DETECTION OF EXIT NUMBER FOR THE BLIND AT THE SUBWAY STATION

Ho-Sub Yoon, Jae Yeon Lee and Eun-Mi Ji 


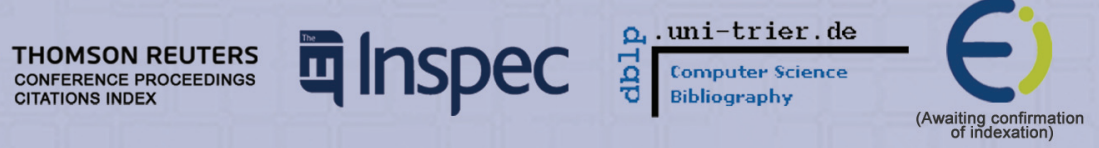

